\title{
The posterior epidural ligament in the thoracic region: a cadaveric and histological study
}

\author{
C.T. Rimmer, P.J. Adds \\ Institute of Medical and Biomedical Education (Anatomy), St. George's University of London, Cranmer Terrace, London, \\ United Kingdom
}

[Received: 1 January 2018; Accepted: 26 February 2018]

\begin{abstract}
The existence of posterior epidural ligaments (PEL) has been established in the lumbar region, but they have hitherto not been shown to exist in the thoracic vertebral column. Their identification is of clinical significance in respect to incidental durotomy and the circulation of cerebrospinal fluid (CSF). Fourteen thoracic spine sections were dissected by cutting through the intervertebral disc and separating the ligamentum flavum from the vertebra above. The dural sheath was gently retracted anteriorly to identify macroscopic connections between the ligamentum flavum and the dura. Macroscopic connections observed were dissected out, retaining some dural sheath and ligamentum flavum. Histological staining with haematoxylin and eosin and Miller's elastin stain was used to investigate cellular connections. Thoracic PELs were positively identified in 5 of the 14 cadavers (35.7\%). Histology showed similarities between the thoracic and lumbar PELs. Fifteen separate PELs were identified within these five thoracic sections. The thoracic PEL has sufficient tensile strength to present a risk to the integrity of the dural sheath during surgery, and surgeons should be aware of these connections when operating on the thoracic spine. PELs may also contribute to the circulation of CSF in the spinal subarachnoid space. (Folia Morphol 2018; 77, 4: 748-751)
\end{abstract}

Key words: thoracic spine, dura, ligamentum flavum, incidental durotomy, cerebrospinal fluid

\section{INTRODUCTION}

The identification of the posterior epidural ligament (PEL) by Connor et al. [4], as distinct from the anterior or lateral Hoffman's ligaments [5, 12], showed that connections exist between the dural sheath and ligamentum flavum in the lumbar spine. Connor et al. [4] described the PEL as a substantial structure connecting the dural sheath to the ligamentum flavum. Such connections between the dura and ligamentum flavum may be implicated in incidental durotomy during posterior approach spinal surgery. Incidental durotomy can lead to other postoperative complications such as the development of pseudomeningocele or arachnoiditis [3]. If this is not noticed, and repaired, at the time of surgery, complications may mean that revision surgery is required [6], and revision surgery itself carries a higher risk of incidental durotomy $[1,11]$. The PEL may also help to support and protect the spinal cord and dural sheath [12], as well as aiding the circulation of cerebrospinal fluid (CSF) in the spinal subarachnoid space, as the thoracic spine is flexed and rotated [13]. The work of Connor et al. [4] showed the presence of the PEL in the lumbar region. The aim of the present study was to investigate the presence, and histological structure, of the PEL in the thoracic spinal region. 


\section{MATERIALS AND METHODS}

Fourteen thoracic segments were available for this study. The spine specimens were removed from formalin-fixed cadavers that had been donated for anatomical examination and research under the $\mathrm{Hu}$ man Tissue Act (2004). There were 10 females and 4 males, average age 84.57 (range 67-92) years. None of the cadavers had any known history of spinal pathology or surgery.

The individual vertebrae were separated using a brain knife, scalpel, blunt dissection and retractors, to gain access to the posterior epidural space of each thoracic vertebra. The cut edge of the dural sheath was gripped with forceps and carefully retracted forward, away from the lamina (Fig. 1). Macroscopic connections between the dura and ligamentum flavum thus revealed were documented and photographed, and dissected out for histological investigation.

Dissected gross connections were re-fixed in 10\% formalin overnight, then processed using a Leica TP1020 Benchtop Tissue Processor (Leica Microsystems Ltd. UK) Wax-embedded specimens were then sectioned transversely at $5 \mu \mathrm{m}$ with a Leica RM2255 microtome (Leica Microsystems Ltd. UK).

Sections were stained with Weigart's haematoxylin and eosin and Miller's elastin stain. These stains allowed the histological structure of the collagen-rich dural sheath, ligamentum flavum and PEL to be visualised and compared, as well as elastin-rich fibres present within the dura, ligamentum flavum and PEL [8].

\section{RESULTS}

Posterior epidural ligaments were identified in 5 of the 14 cadaveric thoracic specimens investigated (35.71\%). Fifteen separate thoracic posterior epidural ligaments connecting the dorsal dura mater to the ligamentum flavum were identified (Fig. 1). The number of PELs tended to increase towards the distal end of the thoracic region, although none was found at T3, T6, T7 and T8. One (7.1\%) PEL was found at each of $\mathrm{T} 1, \mathrm{~T} 2$, T4 and $\mathrm{T} 10 ; 2$ (14.3\%) at T5 and T9; 3 (21.4\%) at $\mathrm{T} 11$, and $4(28.6 \%)$ at T12 (Table 1 ).

Histology confirmed the presence of poorly-differentiated connective tissue between the dura and the ligamentum flavum. The PELs were found to contain a mixture of collagen and elastin fibres, with a lower concentration of elastin than the ligamentum flavum. The histological appearance of the ligament was seen to be distinctly different from the normal anatomy of

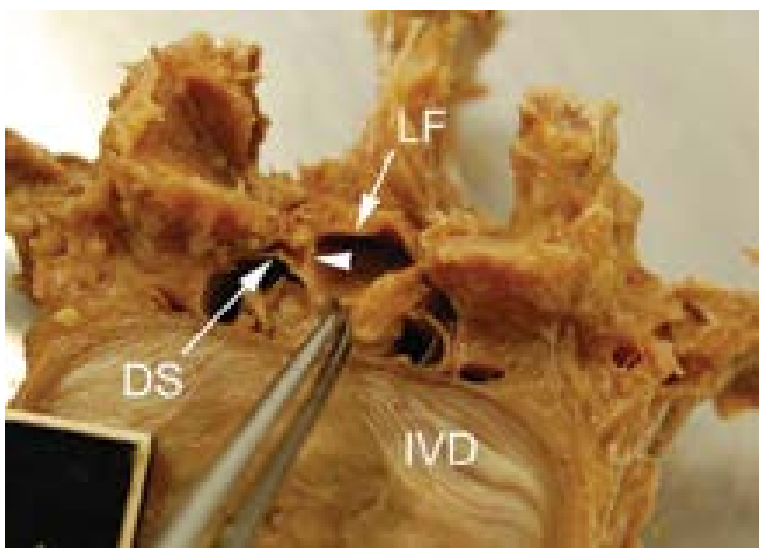

Figure 1. A posterior epidural ligament (arrowhead) at the level of T12. DS — dural sheath; IVD — intervertebral disc; LF — ligamentum flavum.

Table 1. Posterior epidural ligaments (PELs) identified during gross dissection of thoracic vertebral specimens. No PELs were identified at T3, T6, T7 and T8

\begin{tabular}{lcc}
\hline $\begin{array}{l}\text { Thoracic vertebral } \\
\text { level }\end{array}$ & $\begin{array}{c}\text { Number of PELs } \\
\text { observed }\end{array}$ & $\begin{array}{c}\text { Percentage of total } \\
(\%)\end{array}$ \\
\hline T1 & 1 & 7.7 \\
T2 & 1 & 7.7 \\
T4 & 1 & 7.7 \\
T5 & 2 & 15.4 \\
T9 & 2 & 15.4 \\
T10 & 1 & 7.7 \\
T11 & 3 & 23.1 \\
T12 & 4 & 30.8 \\
Total & $\mathbf{1 5}$ & $\mathbf{1 0 0}$ \\
\hline
\end{tabular}

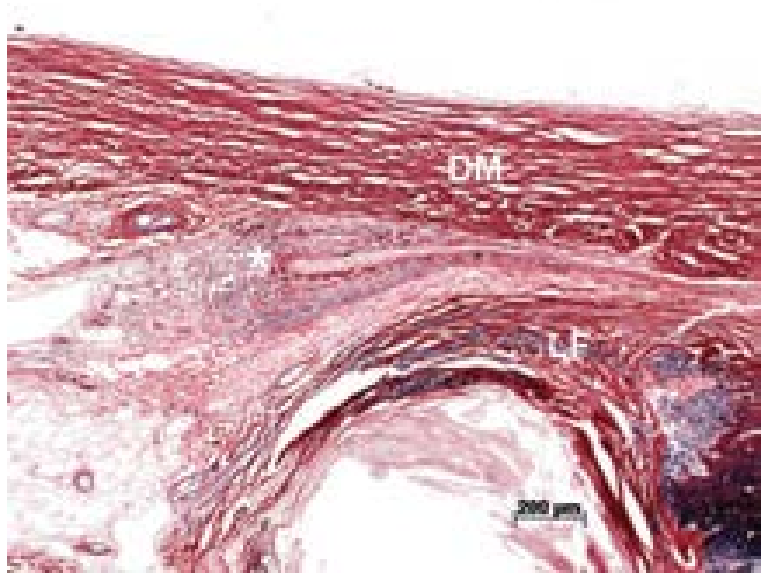

Figure 2. Transverse section, showing posterior epidural ligament (folded) at T11 $\left({ }^{*}\right)$. Miller's elastin stain. DM — dura mater; LF — ligamentum flavum. 


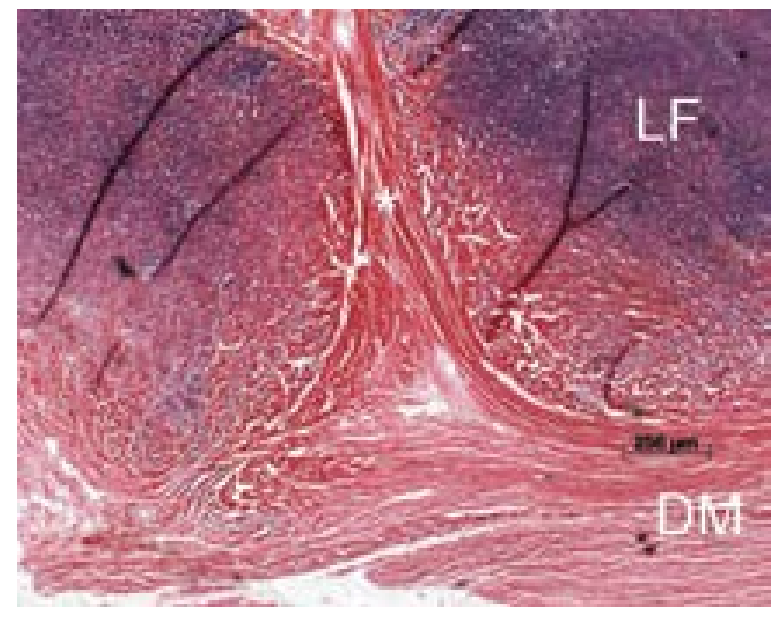

Figure 3. Transverse section, showing posterior epidural ligament $\left.{ }^{*}\right)$ at T12 appearing as a collagenous layer investing the ligamentum flavum (LF); DM — dura mater.

the surrounding structures (Fig. 2). There was some variation in the presentation of the PEL. Two specimens showed the presence of epidural blood vessels within the PEL, and in one specimen, at T12, there was found to be a very close association between the dura mater and the ligamentum flavum, with collagen fibres from the dura mater investing the ligamentum flavum (Fig. 3).

\section{DISCUSSION}

This study has confirmed the presence of the posterior epidural ligament in the thoracic region, using gross inspection and histological analysis. The findings of this study are consistent with those of Connor et al. [4], which showed the presence of the PEL in the lumbar region. Histology on transverse sections confirmed that the structure of the thoracic PEL described here was similar to that of the PEL in the lumbar region. These ligaments consisted of poorly-differentiated, collagen-rich connective tissue, containing variable amounts of elastin, and were distinct from the normal anatomy of both the dural sheath and the ligamentum flavum (Fig. 2). The PEL could also be seen as an investing connection between the dura mater and ligamentum flavum (Fig. 3), and may also contain blood vessels. Elastin fibres were present, but fewer and less densely packed than in the ligamentum flavum. Collagen fibres were also present, but appear as smaller bundles. The similarities between the ligaments described in this study, and those described by Connor et al. [4], suggest that the PEL is a consistent structure, variably present in the posterior epidural space of the lumbar and thoracic regions.
Previously described connections of the posterior epidural space include Blomberg's dorso-median connective tissue band [2], Luyendijk's plica mediana dorsalis [7], and, more recently, the attention to terminal attachment described by Solaroglu et al. [10]. Of these three studies, only Solaroglu et al. [10] included histological analysis. While the attention to terminal attachment shares some characteristics with the PEL, being composed of a mixture of collagen and elastin fibres, it was reported to exist only at the L5 level, and may thus be a case of the PEL being consistently present at the level of $L 5$.

Clinically, the PEL may increase the risk of iatrogenic dural tears during posterior approach spinal surgery, a potentially serious complication [11]. Functionally, the PEL may also play a role in the circulation of CSF. A study by Zheng et al. [13], found a close relationship between the cervical dura mater, the craniovertebral joint and cervical intervertebral joints via a dense fascial band arising from the nuchal ligament. They hypothesised that this "to-be-named ligament" caused movements of the head and neck to directly affect the shape of the dural sleeve, and thereby the subarachnoid space, helping to pump CSF through the spinal canal. The PEL, then, may also aid in the circulation of CSF in the spinal canal in the same way, as the spine rotates, flexes and extends. It is known that the flow of CSF is dependent on body position [9], which tends to support this hypothesis.

\section{CONCLUSIONS}

Gross inspection and histological analysis confirmed the presence of the posterior epidural ligament in the thoracic region. The histological similarities between the connections identified in the lumbar and thoracic spine suggest that the PEL is a consistent feature, variably present in both regions. Further investigation into the distribution of these attachments is needed to increase our understanding of the role and clinical significance of the PEL. Surgeons should be aware of these connections during spinal surgery, particularly during thoracic and lumbar surgeries which require a posterior approach.

\section{Acknowledgements}

The authors gratefully acknowledge the body donors, without whom this study would not have been possible. 


\section{REFERENCES}

1. Benzel EC. Spine Surgery: Techniques, Complication Avoidance and Management, 2nd Edn. Elsevier, Pennsylvania 2005.

2. Blomberg R. The dorsomedian connective tissue band in the lumbar epidural space of humans: an anatomical study using epiduroscopy in autopsy cases. Anesth Analg. 1986; 65(7): 747-752, indexed in Pubmed: 3717614.

3. Chen Z, Shao $P$, Sun $Q$, et al. Risk factors for incidental durotomy during lumbar surgery: a retrospective study by multivariate analysis. Clin Neurol Neurosurg. 2015; 130: 101-104, doi: 10.1016/j.clineuro.2015.01.001, indexed in Pubmed: 25600349.

4. Connor MJ, Nawaz S, Prasad V, et al. The posterior epidural ligaments: a cadaveric and histological investigation in the lumbar region. ISRN Anat. 2013; 2013: 424058, doi: 10.5402/2013/424058, indexed in Pubmed: 25938100.

5. Kimmell KT, Dayoub H, Shakir $H$, et al. Spinal dural attachments to the vertebral column: An anatomic report and review of the literature. Surg Neurol Int. 2011; 2: 97, doi: 10.4103/2152-7806.82990, indexed in Pubmed: 21811703.

6. Koo J, Adamson R, Wagner FC, et al. A new cause of chronic meningitis: infected lumbar pseudomeningocele. Am J Med. 1989; 86(1): 103-104, indexed in Pubmed: 2642652.

7. Luyendijk W. The plica mediana dorsalis of the dura mater and its relation to lumbar peridurography (canalogra- phy). Neuroradiology. 1976; 11(3): 147-149, indexed in Pubmed: 958615.

8. Nakagawa H, Mikawa Y, Watanabe R. Elastin in the human posterior longitudinal ligament and spinal dura. A histologic and biochemical study. Spine. 1994; 19(19): 2164-2169, indexed in Pubmed: 7809748.

9. Orešković $D$, Klarica $M$. A new look at cerebrospinal fluid movement. Fluids Barriers CNS. 2014; 11(16): 1-3, doi: 10.1186/2045-8118-11-16, indexed in Pubmed: 25089184.

10. Solaroglu I, Okutan O, Beskonakli E. The ATA and its surgical importance: a newly described ligament lying between the dural sac and the ligamentum flavum at the L5 level. Spine. 2011; 36(16): 1268-1272, doi: 10.1097/ BRS.0b013e3181f81511, indexed in Pubmed: 21270704.

11. Tafazal SI, Sell PJ. Incidental durotomy in lumbar spine surgery: incidence and management. Eur Spine J. 2005; 14(3): 287-290, doi: 10.1007/s00586-004-0821-2, indexed in Pubmed: 15821921.

12. Wadhwani $S$, Loughenbury $P$, Soames R. The anterior dural (Hofmann) ligaments. Spine. 2004; 29(6): 623-627, indexed in Pubmed: 15014271.

13. Zheng N, Yuan XY, Li YF, et al. Definition of the to be named ligament and vertebrodural ligament and their possible effects on the circulation of CSF. PLoS One. 2014; 9(8): e103451, doi: 10.1371/journal. pone.0103451, indexed in Pubmed: 25084162. 